\title{
Mit Wasser gegen Kopfschmerzen und Migräne
}

Peter W. Gündling

\section{Ätiologie und Klassifikation der Kopfschmerzen}

Kopfschmerzen sind so häufig, dass sie oft gar nicht als Erkrankung wahrgenommen werden. Nach Angaben der Deutschen Migräne- und Kopfschmerzgesellschaft leidet etwa jeder zweite Deutsche regelmäßig an Kopfschmerzen. Etwa jeder Vierte hat Spannungskopfschmerzen, jeder Neunte Migräne und bei höchstens $1 / 4$ der Betroffenen sei je eine zuverlässige Diagnose gestellt worden [4]. Die Internationale Kopfschmerzgesellschaft (IHS) führt in ihrer aktuellen Klassifikation ca. 300 Formen von Kopfschmerzen auf [10].

Ähnlich vielfältig wie die Formen sind auch die Ursachen, deren wichtigste in Kasten 1 aufgeführt sind. Und gerade in den beiden häufigsten Ursachen, muskuläre Verspannungen und vaskuläre Störungen haben Naturheilverfahren im Allgemeinen und die Hydrotherapie im Besonderen einen sehr hohen Stellenwert.

\section{Diagnostik}

Die Diagnose von Kopfschmerzen und Migräne wird in erster Linie klinisch gestellt. Um eventuelle gefährliche Verläufe nicht zu übersehen, ist die genaue Erhebung der Anamnese - ggf. einschließlich eines Kopfschmerztagebuchs - und eine allgemeine körperliche und neurologische Untersuchung unerlässlich. Technische Untersuchungsverfahren werden nur dann erforderlich, wenn aufgrund des klinischen

\section{ZUSAMMENFASSUNG}

Nicht nur bei Herz-Kreislauf-Krankheiten, auch in der Schmerzbehandlung und speziell bei Kopfschmerzen und Migräne stellen hydrotherapeutische Maßnahmen eine wertvolle Option in einer ganzheitlich-naturheilkundlichen Therapie dar. Im Rahmen der Ordnungstherapie eingesetzt verbessert sie - als typisches Regulationsverfahren - die Funktion und Regulation des vegetativen Nervensystems und trägt so zur Normalisierung des Muskeltonus und der Durchblutung bei. Damit können - mit relativ geringem Aufwand - nicht nur die medikamentösen und anderen nicht medikamentösen Maßnahmen ergänzt und verstärkt, sondern auch die Selbsthilfefähigkeit der betroffenen Patienten wirksam unterstützt werden.

\section{Schlüsselwörter}

Hydrotherapie, Kopfschmerz, Migräne, Ordnungstherapie.

Befundes sekundäre Ursachen gezielt ausgeschlossen werden müssen. Dazu zählen z.B. zerebrale Raumforderungen, entzündliche Prozesse oder metabolische Störungen.

Wichtige anamnestische Fragen sind [8]:

- „An wie vielen Tagen im Monat treten die Kopfschmerzen auf?“

- „Wie lange dauern die einzelnen Kopfschmerzperioden?“

- „Wann treten die Kopfschmerzen auf? Gibt es bestimmte Auslöser?"

- „Wie viele unterschiedliche Formen von Kopfschmerzen können Sie unterscheiden?“

\section{ABSTRACT}

Hydrotherapeutic measures are a valuable option in holistic naturopathic therapy for the treatment of cardiovascular diseases as well as for the treatment of pain, especially of headache and migraine. Used within the scope of regulative therapy, hydrotherapy improves - as a typical regulatory procedure - the function and regulation of the vegetative nervous system, thereby contributing to the normalization of muscular tone and blood flow. With hydrotherapy, it is possible - with relatively little effort - not only to complement and strengthen the medicamentous as well as non-medicamentous measures, but also to effectively support the ability of the patients affected to help themselves.

\section{Keywords}

Hydrotherapy, headache, migraine, regulative therapy.

- „Können Sie einen typischen Kopfschmerzanfall schildern?"

- „Können Sie die Schmerzen genau beschreiben?"

- „Welche Begleitsymptome haben Sie?"

- „An wie vielen Tagen pro Monat nehmen Sie Medikamente zur Kopfschmerzbehandlung?“

Damit bekommt man nicht nur einen Hinweis auf die Kopfschmerzform und -ursache, sondern auch auf den Schweregrad der Erkrankung. Die genauen diagnostischen Kriterien der Internationalen Kopfschmerzgesellschaft (IHS) für Migräne ohne Aura sind in Kasten 2 zusammenge- 
stellt. Weitere finden sich auf der Website der IHS [10].

\section{Ganzheitliche Therapie}

Leider wird in aller Regel bei Kopfschmerzen vorrangig zu Schmerzmitteln gegriffen. Abgesehen davon, dass es diese auch auf pflanzlicher und homöopathischer Basis gibt, bedeuten verantwortungsvolles Handeln und ganzheitliche Therapie, dass vorrangig nicht mit medikamentösen, zu Beginn sogar nicht einmal mit therapeutischen, sondern zunächst mit allgemeinen Maßnahmen interveniert werden sollte. Sieht man sich die Ursachen chronischer Erkrankungen an, trifft man neben der genetischen Veranlagung immer wieder auf 3 alte Bekannte: Fehlernährung, Bewegungsmangel und negativen Stress. Diese Auslöser gilt es abzubauen, bevor oder zumindest während man sich spezifischeren Therapiemaßnahmen zuwendet [8].

Das bedeutet bei Migräne z.B. Verzicht auf Nahrungsmittel, die einen Anfall auslösen können wie Glutamat oder auch Tyramin, das u.a. in Käse und Rotwein vorkommt. Das heißt aber auch Neuordnung des Essverhaltens wie regelmäßige Mahlzeiten, gründliches Kauen und frühe, kleine und leicht verdauliche Speisen am Abend sowie regelmäßige Entspannung und mäßige aber regelmäßige Bewegung (10000 Schritte pro Tag) und nicht zuletzt genügend trinken (mind. 1,5-2 l) und genügend Schlaf.

\section{Hydrotherapie nach Kneipp}

Neben diesen 3 Grundpfeilern der klassischen Naturheilkunde kann speziell auch die vierte Säule, die Hydrotherapie, um die sich Sebastian Kneipp besondere Verdienste erworben hat, eine Restrukturierung im Sinne der Ordnungstherapie darstellen [11].

Die besten Ergebnisse erzielen hydrotherapeutische Anwendungen, wenn sie als fester Bestandteil in den Tagesablauf integriert werden. So lassen sich z. B. kalte Gesichts- und/oder Armgüsse sehr schnell und einfach in den morgendlichen Badezimmerbesuch und das warme oder Wechselfußbad als wohltuende Entspannungseinheit am Abend einplanen.

In diesem Sinne kann praktisch jede korrekt ausgeführte und richtig dosierte Wasseranwendung die Regulation des vegetativen Nervensystems und damit den Tonus der Muskulatur und der Blutgefäße positiv beeinflussen und bei regelmäßiger Anwendung mittel- und langfristig zur Verringerung der Kopfschmerzhäufigkeit und -intensität beitragen. Speziell bei Migräne bewirken regelmäßige Kneipp'sche Wasseranwendungen eine Stabilisierung überschießender vasomotorischer Reaktionen [2].

\section{Allgemeine Wirkungen}

Kaltes wie auch warmes Wasser stimuliert die äußeren Thermorezeptoren, die diese Information über afferente (Aס-) Nervenfasern an das zentrale Thermoregulationszentrum im Hypothalamus weiterleiten. Über motorische und vegetative Fasern kommt es nun je nach thermischem Reiz durch Beeinflussung der Skelettmuskulatur und der inneren Organe zur Wärmebildung und Dilatation oder (kurzzeitiger) Konstriktion der Gefäße. Zudem werden immunologische und hormonell-humorale Reaktionen insbesondere des Hypophysen-Nebennierenrinden-Systems angeregt [3].

Je nach Dauer und Intensität dieser Reizfaktoren sowie der Größe des behandelten Körperareals kommt es durch das Wasser einerseits zur Steigerung der Durchblutung und der Stoffwechselleistung des behandelten Gebietes und seiner Reflexzonen [5]. Andererseits erfolgt aber auch eine Freisetzung von Mediatoren, Aktivierung von Lysozymen und anderen Enzymen, Anregung der lokalen und allgemeinen Abwehrmechanismen, Erhöhung der Schmerzschwelle sowie einer Erhöhung der Dehnbarkeit des Bindegewebes und Verminderung des Muskeltonus [2].

\section{Wirkungen auf das Vegetativum bei Kopfschmerzen}

Erhöhter Muskeltonus und daraus folgende Spannungskopfschmerzen ebenso wie Migräne sind Ausdruck eines überaktiven Sympathikus. Hydrotherapeutische An-
Kasten 1

\section{Ätiologie von Kopfschmerzen}

1. Vaskuläre Ursachen

a. Migränetyp

b. Nichtmigränoider Typ

- allgem. zerebrovaskuläre Prozesse (Arteriosklerose, Hypertonie)

- Allgemeininfektionen (Viren, Leptospiren, Rickettsien)

- metabolische, endokrine Reaktionen (Hypoglykämie, Hypothyreose)

- toxische Reaktionen (Nikotin, Blei, Kohlenmonoxid, Benzin, Nitrite)

- posttraumatisch, postkonvulsiv

- Medikamentenentzug

2. Entzündliche Prozesse

- Meningitis, Enzephalitis (viral, bakteriell)

- Arteriitis, Phlebitis

- Augen, Zähne, Kiefer, HNO, Gelenke

3. Mechanische Einwirkungen

- primäre/sekundäre Tumoren (M. Cushing, Akromegalie)

- Abszesse, Hämatome, Subarachnoidalblutung

- Hydrozephalus, Hirndruck

- Gelenk-, Bänder-, Muskeldysfunktion

4. Trauma

- Skalp, Knochen

- intrakranielle Prozesse

- HWS-Dysfunktion

5. Neuralgien

6. Psychogene Faktoren

wendungen haben bereits durch die Stimulation der Thermorezeptoren einen Effekt auf das vegetative Nervensystem und das Hypophysen-Nebennierenrinden-System und können so bei wiederholter Anwendung dazu beitragen, dass es zu einer Regulationsverbesserung und einem Ausgleich kommt. Werden diese Reize regelmäßig appliziert, erfolgt ein Anpassungseffekt, bei dem es durch die Downregulation von Adrenalin zu einer dauerhaften Minderung des Sympathikotonus und einer Regeneration des Regelmechanismus kommt. Durch das regelmäßige Gefäßtraining wird die Vasomotion gefördert 


Kasten 2
Diagnostische IHS-Kriterien
der Migräne ohne Aura
A) Mindestens 5 Attacken, die die
Kriterien B - D erfüllen.
B) Kopfschmerzattacken, die (unbehan-
delt oder erfolglos behandelt) 4-72
Stunden anhalten
C) Der Kopfschmerz weist mindestens 2
der folgenden Charakteristika auf:
1. Einseitige Lokalisation
2. Pulsierender Charakter
3. Mittlere oder starke Schmerz-
intensität
4. Wird durch körperliche Routineakti-
vitäten (z. B. Gehen oder Treppen-
steigen) verstärkt oder führt zu de-
ren Vermeidung
D)Während des Kopfschmerzes besteht
mindestens eines:
1. Übelkeit und/oder Erbrechen
2. Photophobie und Phonophobie
E) Nicht auf eine andere Erkrankung
zurückzuführen

und die Endothelfunktion regeneriert [5].

\section{Grundregeln der Hydrotherapie}

Obgleich die Durchführung hydrotherapeutischer Maßnahmen recht einfach ist, müssen doch einige Grundsätze beachtet werden, bei deren Verletzung die Therapie nicht nur nicht wirkt, sondern sogar schaden kann. Das Wohlbefinden des Patienten ist einer der wichtigsten Parameter nach einer richtig dosierten Wasseranwendung. Herzklopfen nach einem Warmvollbad oder anhaltendes Kältegefühl nach einer kalten Anwendung sind Fehlreaktionen, die es durch eine korrekt gewählte und dosierte Anwendung zu vermeiden gilt.

Jeglicher Kaltreiz sollte nur am warmen Körper und auf warmer Haut erfolgen. Ist der Patient oder der entsprechende Körperteil kalt, muss zuvor eine Erwärmung, aktiv durch Bewegung oder passiv durch warme Getränke, warmes Wasser oder Kleidung oder Bettwärme erfolgen. Das gleiche gilt nach einer Anwendung: Wiedererwärmung, aktiv oder passiv [2]. Auch sollten verschiedene Anwendungen i.d. R.
Kasten 3

\section{Prinzipien der Wasserheilkunde [6]}

- Nie kaltes Wasser auf kalte Haut.

- Vor- und nachwärmen.

- Der mildeste Reiz, der wirkt, ist der beste.

- (Wechsel-) Bäder

- Anregung von Stoffwechsel und Durchblutung

- Stabilisierung des vegetativen Nervensystems

- (Wechsel-) Güsse

- Verbesserung der Durchblutung

- Ausgleich des vegetativen Nervensystems

- ausleitend und tonisierend

- Heusack, Moorpackung

- Entspannung der Muskulatur

- Verbesserung der Durchblutung

- Badezusätze

- wärmend, durchblutungsanregend: Ingwer, Senfmehl

- entspannend, vegetativ stabilisierend: Melisse, Lavendel, Baldrian

nicht direkt aufeinanderfolgen, da sie die Reaktionsfähigkeit des Körpers sonst überfordern. Auch unmittelbar vor oder nach den Mahlzeiten sollten - mit Ausnahme von verdauungsfördernden Maßnahmen wie einer feuchtwarmen Abdominalpackung - keine Wasseranwendun-

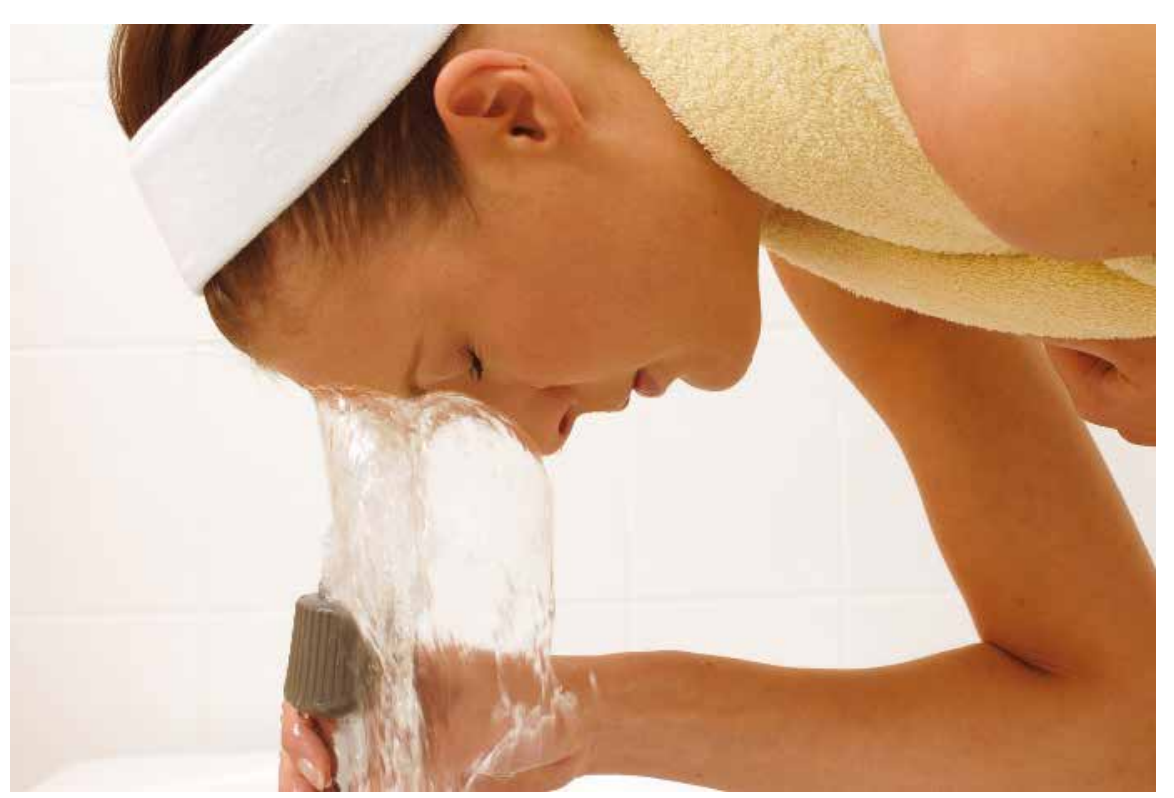

Abb. 1: Der kalte Gesichtsguss wirkt reflektorisch auf die gesamte Kopfregion. @ C Thomas Möller/TVG; nachgestellte Situation

gen durchgeführt werden [1]. Der Zeitabstand sollte mindestens 30-60 min betragen. Schließlich betont bereits Kneipp, dass der mildeste Reiz, der eben noch wirkt, der beste sei. Der häufigste Fehler, der bei der Anwendung von Wasserheilverfahren gemacht würde, sei der zu starke Reiz [11].

Hydrotherapeutische Anwendungen gegen Kopfschmerzen und Migräne Hydrotherapeutische Anwendungen wirken sich ganz allgemein positiv auf das vegetative Nervensystem, den Muskeltonus und die Durchblutung aus. Zur Behandlung von Kopfschmerzen und Migräne im Speziellen haben sich dabei insbesondere Verfahren bewährt, die vom Patienten auch zu Hause leicht selbst durchzuführen sind.

\section{Güsse}

Güsse sind das Herzstück der Kneipp'schen Hydrotherapie und werden mit einem Schlauch von 1,5 m Länge und 3/4-Zoll (20 mm) Durchmesser, einem Gießhandstück, das anstelle des Brausekopfes auf den Duschschlauch geschraubt wird oder im einfachsten Fall einer weichgestellten Handbrause durchgeführt.

Die bei Kopfschmerzen und Migräne angewendeten Formen sind sog. Flachgüsse, die ohne Druck verabreicht werden, sodass das Wasser den jeweiligen Körperteil 
„ummantelt". Dabei unterscheidet man kalte (bis $15^{\circ} \mathrm{C}$ ), temperierte $\left(18-22^{\circ} \mathrm{C}\right.$ ), ansteigende (von ca. $34^{\circ} \mathrm{C}$ bis ca. $44^{\circ} \mathrm{C}$ ) und Wechselgüsse, die zuerst warm (36$38^{\circ} \mathrm{C}$ ), dann kalt und dann nochmals warm und abschließend kalt ausgeführt werden. Diese Anwendungen sind leicht erlernbar und können nach entsprechender Anleitung von jedem selbst ausgeführt werden.

\section{Prinzipien bei der Durchführung von Güssen}

- Die Stärke des Wasserstrahls wird so eingestellt, dass bei schräg nach oben gehaltener Schlauchöffnung, das Wasser etwa handbreit heraussprudelt.

- Der Raum muss gut warm sein.

- Auf Wiedererwärmung durch Bewegung oder im Bett (ggf. mit Socken) achten!

- Der zeitliche Abstand zu körperlichen Anstrengungen sollte mind. $30 \mathrm{~min}$ betragen.

- Bei der Durchführung von Güssen an den Beinen sollte der Patient möglichst auf einem Kunststoff- oder Holzrost stehen, damit die Füße nicht kalt werden.

- Immer herzfern - rechts, unten, außen - beginnen!

- Bei kalten Güssen vorher einatmen und mit Beginn des Gusses ausatmen. Auf ruhige Atmung und entspannte Körperhaltung während des Gusses achten [9].

\section{Kalter Gesichtsguss}

Der von Kneipp als „Schönheitsguss” bezeichnete kalte Gesichtsguss wirkt reflektorisch auf die gesamte Kopfregion und ist sowohl bei Kopfschmerzen als auch bei Erschöpfungszuständen und nachlassender Sehkraft indiziert. Er wird durchgeführt, indem man den Wasserstrahl am nach vorne gebeugten Kopf zunächst von der rechten Schläfe beginnend über die Stirn zur linken Schläfe führt und einige Male hin und her bewegt. Anschließend wird erst die rechte, dann die linke Gesichtshälfte in Auf- und Abwärtsbewegungen und zum Schluss das Gesicht kreisförmig umrundend begossen. Will man es sich einfach machen, kann man auch zu-

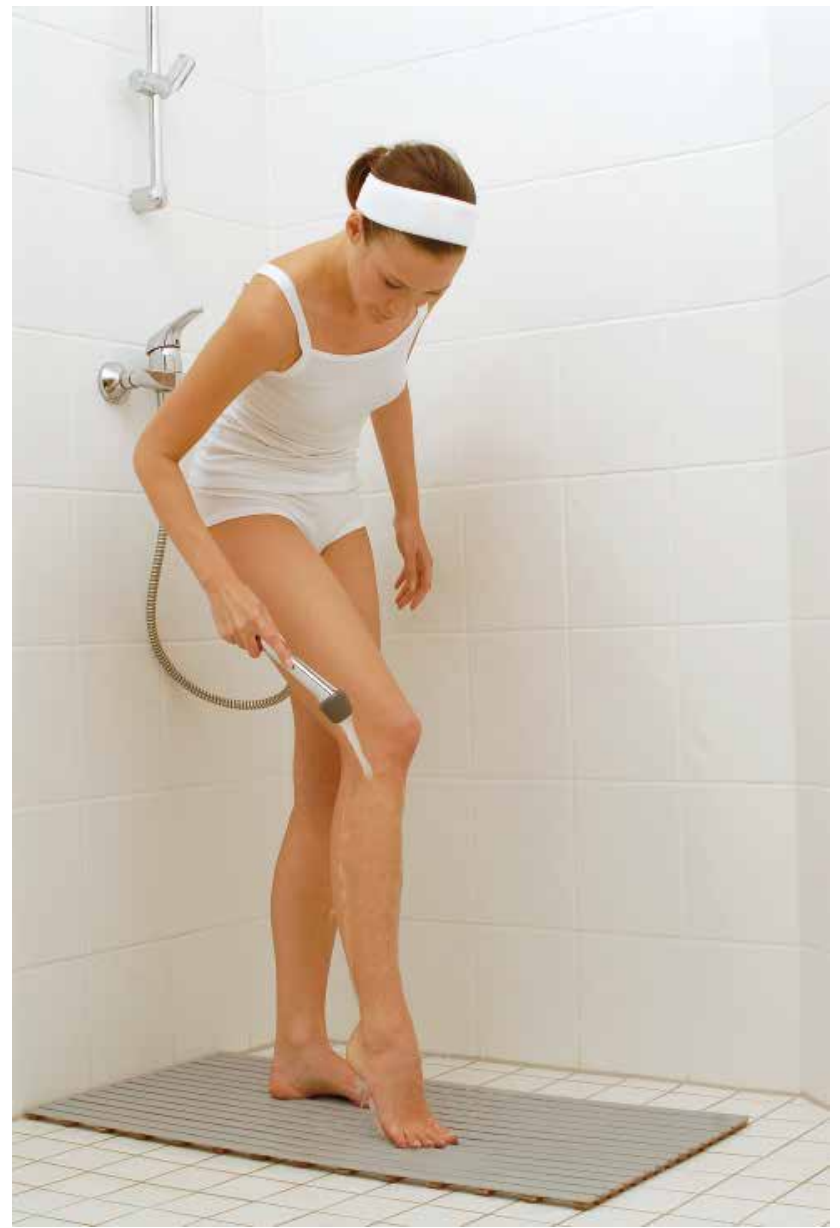

Abb. 2: Der Knieguss ist einfach und schnell durchführbar und ist bei gefäßbedingten Kopfschmerzen indiziert. (c) Thomas Möller/TVG; nachgestellte Situation

nächst die Stirn, dann die Augen und schließlich das ganze Gesicht und die laufende Wasserleitung halten (Abb. 1). Danach soll das Wasser vom Gesicht nur leicht abgetupft werden [2].

\section{Kalter Knieguss, Wechsel-Knieguss} Ebenfalls schnell und einfach durchführbar und indiziert bei gefäßbedingten Kopf- schmerzen ist der Knieguss, der je nach Wärmebedarf kalt oder als Wechselguss durchgeführt werden kann. Er regt die Durchblutung an, erweitert die Arterien, wirkt blutdrucksenkend, entstauend, vegetativ beruhigend und schlaffördernd. Nicht durchführen sollte man ihn bei niedrigem Blutdruck, Menstruation, Ischialgie, sowie Nieren- und Blasenleiden. 


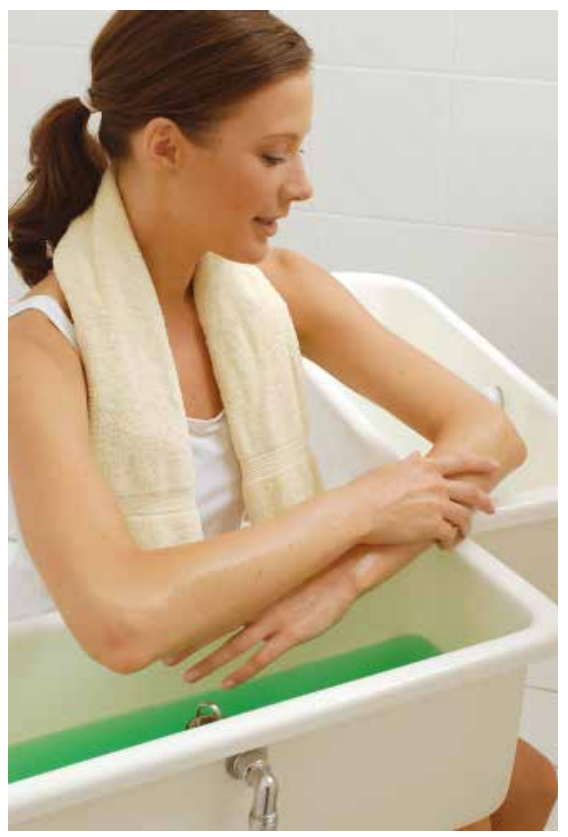

Abb. 3: Beim temperaturansteigenden Armbad kommt man mit nur einem Gefäß aus, benötigt allerdings etwas Zeit. (c) Thomas Möller/TVG; nachgestellte Situation

Durchgeführt wird der kalte Knieguss mit kaltem Wasser bis $15^{\circ} \mathrm{C}$ und er dauert nur etwa $2 \mathrm{~min}$. Dabei beginnt man am rechten lateralen Zehenrand und führt den Wasserstrahl über den Fußrücken außen am Bein nach oben bis eine Hand breit übers Knie, verweilt hier einen Moment und führt den Wasserstrahl dann am inneren Unterschenkel wieder abwärts bis zum Fuß (Abb. 2). Anschließend folgt das linke Bein in gleicher Weise. Ganz zum Schluss folgen die Fußsohlen, erst rechts, dann links.

Im Unterschied dazu beginnt der Wechsel-Knieguss mit $36-38^{\circ} \mathrm{C}$ warmem Wasser, das in gleicher Weise zuerst am rechten, dann am linken Bein vom Fußrücken aufwärts bis übers Knie verabreicht wird. Dieser Warmanteil wird durchgeführt, bis gute Durchwärmung eintritt, erst dann geht es auf der Innenseite wieder abwärts. Danach folgt der Kaltanteil wie oben beschrieben. Warm- und Kaltanteil werden noch einmal wiederholt und zum Schluss beide Fußsohlen kalt abgegossen [1].

\section{Ansteigender Nackenguss}

Eine wahre Wohltat für verspannte und schmerzhafte Muskeln speziell im Rückenund Nackenbereich sowie bei Spannungskopfschmerzen sind (temperatur-) anstei- gende Güsse. Diese wirken entspannend, entkrampfend und durchblutungssteigernd auf das behandelte Muskelareal und reflektorisch auf die segmental zugeordneten Brust-, Bauch- und Beckenorgane. Dadurch werden diese Gebiete wieder besser mit Sauerstoff versorgt, abgelagerte Stoffwechselschlacken abtransportiert und damit die schmerzauslösenden Ursachen beseitigt. Allgemeine Kontraindikationen sind akute Entzündungen im zu behandelnden Bereich. Ist eine entsprechende physikalische Einrichtung vorhanden, kann die Anwendung dort durchgeführt werden. Andernfalls kann auch der Patient angeleitet werden, diese Anwendung zu Hause selbst durchzuführen [8].

Für den Nackenguss sollte sich der Patient am besten mit dem Oberkörper über die Badewanne beugen, während eine zweite Person den weichen Wasserstrahl im Bereich der unteren Hals- und oberen Brustwirbelsäule langsam hin und her, auf und ab bewegt. Die Wassertemperatur sollte anfangs etwa $34^{\circ} \mathrm{C}$ betragen, also weder als warm noch als kalt empfunden werden und ganz langsam, im Verlauf von 10-15 min, immer wärmer bis zur Verträglichkeitsgrenze von etwa $43^{\circ} \mathrm{C}$ hochreguliert werden. Dadurch ist die Wärmeaufnahme des Körpers wesentlich größer, als wenn das heiße Wasser direkt auf die Haut appliziert würde und der Effekt dementsprechend deutlich besser. Die Anwendung wird so lange durchgeführt, bis eine kräftige Rötung (Hyperämie) erreicht ist. Anschließend sollte sich der Patient gründlich abtrocknen und in entspannter Haltung (Unterschenkel erhöht) ruhen. Kontraindiziert ist dieser Guss bei arterieller Hypertonie, Herzinsuffizienz NYHA III und IV, Hyperthyreose, Glaukom und Katarakt [2].

\section{Bäder}

Bei Kopfschmerzen und Migräne sind v.a. warme Bäder angezeigt, die eine vagotonisierende Wirkung haben. Sie dilatieren die Hautgefäße, sodass die Blutmenge in der Haut auf bis zu 1,5l steigt. Die Schweißsekretion steigt, was zur Eindickung des Blutes führt und über eine Sogwirkung zur Entschlackung der Körperzellen und des Zwischenzellraumes führt. Zudem wird die Darmperistaltik aktiviert.
Demgegenüber beeinflussen kalte Bäder den Sympathikus. Sie wirken blutverdünnend, pH-Wert-senkend, blutzuckersteigernd und hemmen die Darmperistaltik.

Darüber hinaus kann diese physikalische Wirkung von Bädern durch den Zusatz bestimmter Substanzen verstärkt werden. Da die Haut imstande ist, die aus Badezusätzen freigesetzten Substanzen aufzunehmen, werden dabei im Blut ähnlich hohe Wirkspiegel wie bei oraler Aufnahme erreicht. Je nach Zusatz und Temperatur können Bäder entspannend oder anregend wirken und fördern so das körperliche und seelische Wohlbefinden.

\section{Warmes Fußbad, Wechselfußbad}

Als Ausdruck vegetativer Fehlregulation im Allgemeinen und Störung des Gefäßtonus im Besonderen leiden nahezu alle Migränepatienten an kalten Füßen. Deshalb können warme und Wechselfußbäder oft wahre Wunder wirken. Besonders bewährt bei Patienten mit Migräne und chronisch kalten Füßen haben sich 38$40^{\circ} \mathrm{C}$ warme Fußbäder über $10-20 \mathrm{~min}$, denen man etwa $4 \mathrm{EL}$ Ingwerpulver zusetzt. Der Ingwerzusatz verstärkt den Wärmeeffekt und wirkt sich so auf den gesamten Körper aus.

Bei beginnender akuter Migräne empfehlen sich warme Fußbäder, denen 4 EL Senfmehl zugesetzt werden. Diese üben einen starken Reizeffekt auf die Haut an den Füßen aus und führen so zu einer Gegenregulation, die die Migräne stoppen kann.

Für das Wechselfußbad werden 2 Gefäße, eines mit etwa $38^{\circ} \mathrm{C}$ warmen und eines mit kaltem Wasser und etwa $10 \mathrm{~min}$ Zeit benötigt. Begonnen wird mit dem Warmbad, in das beide Füße etwa 5 min lang hinein gestellt werden. Anschließend folgt etwa $15 \mathrm{~s}$ lang das Kaltbad. Danach wiederholt man beide Teile, 5 min lang warm und $15 \mathrm{~s}$ lang kalt, streift das Wasser ab und fühlt schnell einen wohltuenden Effekt. Zur Wirkungsverstärkung können dem warmen Wasser wieder Badezusätze wie Rosmarinextrakt oder Ingwerpulver zugegeben werden.

Dabei ist zu beachten, dass bei Fußbädern nicht nur die Füße, sondern möglichst die gesamten Unterschenkel bis knapp unter die Knie im Wasser stehen. 
Hierfür benötigt man tiefere Wannen, die im Fachhandel erhältlich sind. Zur Not tut es auch ein sauberer 20-Liter Eimer. Ausnahme sind Patienten mit ausgeprägten Krampfadern. Diese sollen tatsächlich nur die Füße bis zum Knöchel in das warme Wasser stellen.

Kaltes Armbad, Wechselarmbad, temperaturansteigendes Armbad

Ähnlich wie mit Fußbädern kann man mit einem Armbad verfahren, das auch im täglichen Wechsel mit einem Fußbad durchgeführt werden kann. Am einfachsten durchzuführen ist das kalte Armbad, für das schon eine Wanne oder ein tieferes Waschbecken mit möglichst kaltem Wasser reicht. Hier werden beide Arme für etwa 30 s möglichst weit eingetaucht, abgestreift und für Wiedererwärmung gesorgt [9] (Abb. 3).

Mit ebenfalls nur einem Gefäß kommt das temperaturansteigende Armbad aus. Allerdings werden dafür etwas mehr Zeit und eine Wasserablaufmöglichkeit benötigt. Es beginnt mit einer indifferenten Temperatur von $33-34{ }^{\circ} \mathrm{C}$ und wird durch langsames Zulaufen von heißem Wasser innerhalb von $15-20 \mathrm{~min}$ auf etwa $42{ }^{\circ} \mathrm{C}$ erhöht. Dies führt zu einer besonders schonenden Vasodilatation und ist sowohl bei Herz-Kreislauf-Krankheiten als auch bei vasomotorischen Kopfschmerzen angezeigt. Speziell hierbei ist eine mindestens 20-minütige Nachruhe wichtig [2].

\section{Heublumensack}

Als letzte hydrotherapeutische Anwendung sei noch auf die entspannende und schmerzlindernde Wirkung von mit Wasserdampf erwärmten Heublumensäcken hingewiesen (Abb. 4). Sie werden auch als das Morphium der Naturheilkunde bezeichnet und sind u. a. bei Spannungskopfschmerzen indiziert. Dazu füllt man ein etwa $25 \times 50 \mathrm{~cm}$ großes Leinensäckchen $z u ~ 2 / 3$ mit Heublumen (oder kauft ein entsprechendes Fertigprodukt) und erwärmt es etwa 20 min lang über einem Topf mit kochendem Wasser und Siebeinsatz. Anschließend legt sich der Patient vorsichtig (heiß!) mit dem Schulter-Nacken-Bereich auf die Wärmepackung, wird zusätzlich mit 1-2 Decken gut eingepackt und bleibt so etwa 45 min liegen, bis der Heusack ab-

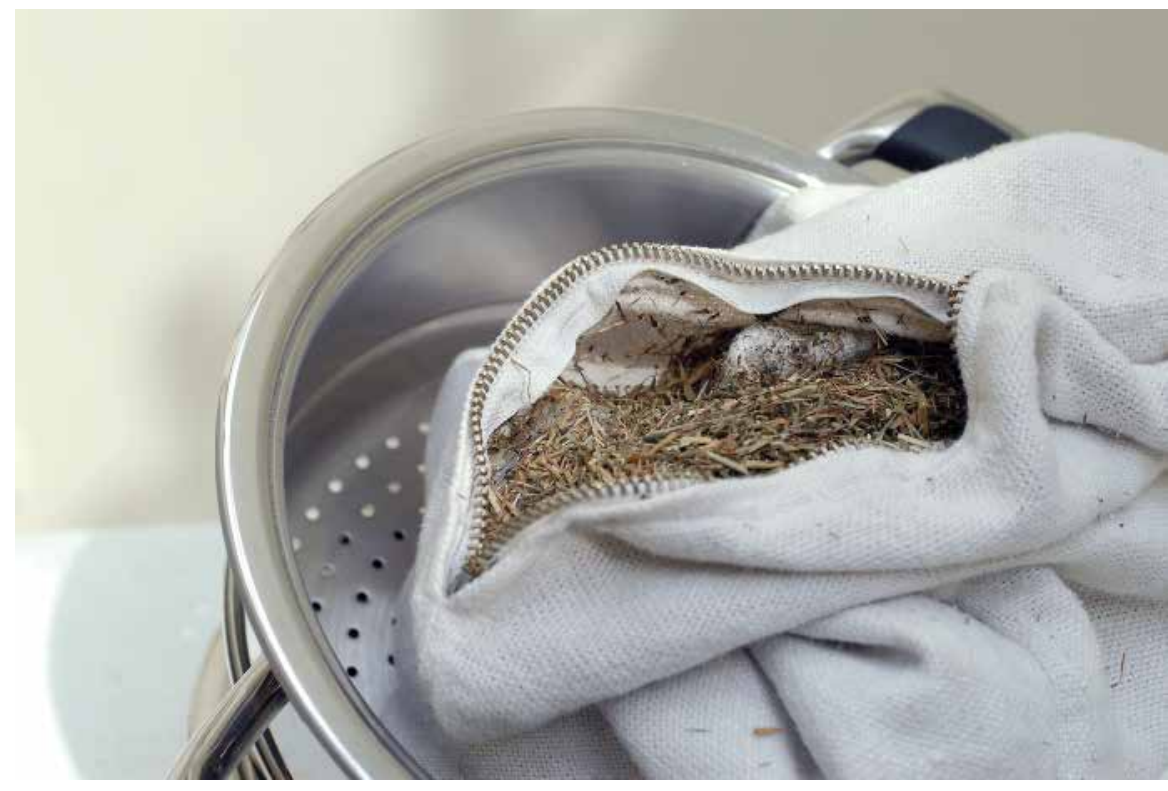

Abb. 4: Das Morphium der Naturheilkunde: der Heublumensack wirkt entspannend und schmerzlindernd. (c) Thomas Möller/TVG

gekühlt ist. Die intensive Wärme wirkt stark vagotonisierend und führt so zu Muskelentspannung und Vasodilatation.

\section{Einlauf}

Eine Sonderform der Hydrotherapie sind Einläufe, die in akuten Fällen auch ohne die anderen Maßnahmen zur Schmerzlinderung beitragen können. So unbeliebt der Einlauf auch sein mag, so hilfreich ist er jedoch. Er ist kostengünstig und einfach und kann auch vom Patienten selbst durchgeführt werden. Benötigt wird dazu lediglich ein Einlaufgerät, ein Irrigator oder Klyso.

Der Irrigator ist ein Kunststoffgefäß, ähnlich einem Messbecher mit einem Gummischlauch, das mit lauwarmem Wasser, i.d.R. ohne jegliche Zusätze, gefüllt wird. Das Einführstück wird mit etwas Salbe eingestrichen und in den Enddarm eingeführt. Dabei kann der Patient liegen oder auf der Toilette sitzen. Dann wird das Gefäß in Schulterhöhe gehalten und das Wasser in den Dickdarm einlaufen lassen.

Die Durchführung mit dem Klyso, einem Gummischlauch mit einem Pumpball in der Mitte, funktioniert im Prinzip genauso. Nur wird das Wasser aus einem Gefäß, z.B. einem Waschbecken, in den Darm gepumpt und taugt eher für die Selbstbehandlung. Hat der Patient das Gefühl, dass der Darm voll ist, zieht man das
Einführstück heraus und der Patient lässt das Wasser direkt auf der Toilette aus dem Darm heraus. Dieses Vorgehen kann noch 1- bis 2-mal wiederholt werden, bis der gesamte Dickdarm ausgespült ist. Auf diese Weise kommt es zur Ausleitung von Krankheitsgiften, die durch Fäulnis- und Gärungsprozesse entstanden sind und zu Entzündungen und Autointoxikation führen. Diese führt nicht nur zu lokalen und regionalen Schmerzen, sondern bewirkt auch Regulationsstörungen, die Schmerzzustände wie die Migräne verursachen [7].

\section{Ein Fall aus der Praxis}

\section{Anamnese und Untersuchung}

Eine schlanke 37-jährige Büroangestellte sucht meine Praxis auf und klagt über rezidivierende Migräneanfälle alle 1-2 Wochen mit einer Dauer von bis zu 3 Tagen sowie zwischendurch noch Spannungskopfschmerzen. Sie habe schon alles Mögliche probiert, u. a. verschiedene Medikamente, auch Akupunktur und manuelle Therapie, was ihr jeweils nur kurzzeitig geholfen habe. Sie ernähre sich überwiegend vegetarisch, habe genügend Schlaf, sei aber übergenau. Auf Nachfragen gibt sie an, dass sie häufig einen aufgeblähten Leib und harten Stuhlgang habe und wohl zu wenig trinke. 
Bei der Untersuchung finden sich Verspannungen und mehrere bis haselnussgroße, scharfkantig begrenzte Verhärtungen im Nackenbereich und kalte Hände und Füße. Der gesamte Abdominalbereich ist gestaut und verhärtet.

\section{Therapie}

Ich erkläre der Patientin die Zusammenhänge von Verdauungstrakt, Autointoxikation und Migräne und empfehle ihr eine Intensivdiät nach Dr. F.X. Mayr. Diese lehnt die Patientin jedoch aus beruflichen Gründen $a b$, willigt jedoch ein, ihre Ernährung neu zu ordnen, mehr zu trinken und gründlicher zu kauen. Zudem erklärt sie sich bereit, täglich ein Wechselarm- oder Fußbad zu machen. Begleitend dazu führe ich mit ihr eine Neuraltherapie durch. Für den Akutfall empfehle ich ihr die Durchführung von Fußbädern mit Senfmehl und Einläufe.

\section{Verlauf}

Unter dieser Therapie verringern sich im Laufe der folgenden 3 Monate die Kopfschmerzen und die Migräne tritt nur noch alle 3-4 Wochen auf. Auch klingen die Migräneanfälle durch die Einläufe sehr viel schneller ab. Zwischenzeitlich sind 2 Jahre vergangen und die Patientin hat vor einigen Monaten doch noch eine F.X. MayrKur durchgeführt. Nun hat sie kaum noch Kopfschmerzen. In den letzten Monaten hatte sie nur eine kleine Migräneattacke, die sie aber mit dem Fußbad schnell in Griff bekam.
Interessenkonflikte: Der Autor erklärt, dass keine wirtschaftlichen oder persönlichen Verbindungen bestehen.

\section{Online zu finden unter:}

http://dx.doi.org//10.1055/s-0033-1357716

\section{Literatur}

[1] Bachmann RM, Schleinkofer GM. Die Kneipp-Wassertherapie. Stuttgart: Thieme; 1992

[2] Brenke R, Polonius D. Hydro- und Thermotherapie. In: Melchart D, Brenke R, Dobos $G$ et al., Hrsg. Naturheilverfahren. Stuttgart: Schattauer; 2002: 294-331

[3] Brenke R, Conradi E. Hydrotherapie. In: Kraft K, Stange R, Hrsg. Lehrbuch Naturheilverfahren. Stuttgart: Hippokrates; 2010: 181-208

[4] Deutsche Migräne- und Kopfschmerzgesellschaft. Im Internet: www.dmkg.de

[5] Fiscus KA, Kaminski TW, Powers ME. Changes in lower-leg blood flow during warm-, cold-, and contrast-water therapy. Arch Phys Med Rehabil 2005; 86 (7): 1404-1410

[6] Gündling PW. Brennpunkt Herz. Natürlich heilen und vorbeugen bei Herz-Kreislauf-Problemen. Baden-Baden: Aurelia; 2004

[7] Gündling PW. Ausleitende Verfahren in der Schmerztherapie. Biologische Medizin 2004; 33 (2): 61-65

[8] Gündling PW. Die komplementäre Behandlung von Kopfschmerzen und Migräne. Die Naturheilkunde 2010; 87 (4): 22-25

[9] Gündling PW. Hydrotherapie bei Hypertonie. EHK 2014; 63 (1): 5-13

\section{ÜBER DEN AUTOR}

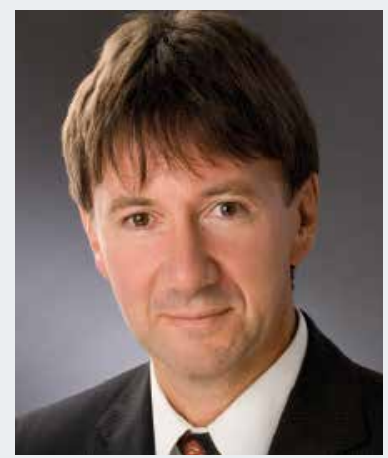

Peter W. Gündling ist seit 1988 in eigener Praxis niedergelassen. Seit 2006 Professor für Naturheilkunde und komplementäre Medizin an der Hochschule Fresenius in Idstein, wo er als Studiendekan ein neues Masterstudium zur Naturheilkunde und komplementären Medizin für Ärzte entwickelt hat und leitet.

KORRESPONDENZADRESSE

Prof. Dr. med. Peter W. Gündling MME M.Sc. Arzt für Allgemeinmedizin, Naturheilverfahren, Akupunktur, Chirotherapie, Sportmedizin, Ernährungsmediziner, Badearzt

Studienleitung Naturheilkunde und komplementäre Medizin

Hochschule Fresenius Idstein Lehrbeauftragter der Johann-Wolfgang-Goethe-Universität Frankfurt a. Main

Sebastian-Kneipp-Straße 4 65520 Bad Camberg/Ts.

E-Mail: guendling@hs-fresenius.de

[10] Headache Classification Committee of the International Headache Society (IHS). The International Classification of Headache Disorders. 3rd ed. (beta version). Cephalgia 2013; 33 (9) 629-808

[11] Kneipp S. Meine Wasser-Kur. 50. Aufl. Kempten: Kösel; 1888 